\title{
Variabilité phénotypique des sorghos locaux de deux régions du Burkina Faso : la Boucle du Mouhoun et le Centre-Ouest
}

Clarisse Pulchérie

Barro-Kondombo ${ }^{1}$

Kirsten Vom Brocke ${ }^{2}$

Jacques Chantereau ${ }^{2}$

Fabrice Sagnard ${ }^{3}$

Jean-Didier Zongo 4

${ }^{1}$ Institut de l'environnement

et des recherches agricoles (Inera),

Centre régional de recherches

environnementales et agricoles (CRREA)

du Centre,

Saria BP 10,

Koudougou

Burkina Faso

<clarissebk@yahoo.fr>

${ }^{2}$ Centre de coopération internationale en recherche agronomique

pour le développement (Cirad),

Unité propre de recherche (UPR)

"Agrobiodiversité des plantes de savanes",

Avenue Agropolis

TA A-08/01,

34398 Montpellier cedex 5

France

<kirsten.vom_brocke@cirad.fr>

<jacques.chantereau@cirad.fr>

${ }^{3}$ Centre de coopération internationale

en recherche agronomique

pour le développement (Cirad),

Unité propre de recherche (UPR) « Gestion

des ressources génétiques et dynamiques

sociales"

Campus CNRS/CEFE,

1919 , route de Mende,

34293 Montpellier cedex 5

France

<fabrice.sagnard@cirad.fr>

${ }^{4}$ Unité de formation et de recherche en sciences de la vie et de la terre (UFR/SVT),

Université de Ouagadougou,

03 BP 7021

Ouagadougou 03

Burkina Faso

<zongojd@univ-ouaga.bf>

\begin{abstract}
Résumé
La classification raciale de 190 variétés locales de sorgho prospectées dans deux régions du Burkina Faso et évaluées pour 27 descripteurs agromorphologiques fait valoir une prépondérance de Guinea (96,8 \% des variétés collectées) et une faible présence de Durra (1,6\%) et Caudatum $(0,5 \%)$ tandis que le reste $(1,1 \%)$ des variétés locales est resté non classé. Parmi les sorghos Guinea, il y a une majorité de gambicum et quelques margaritiferum qui présentent une large gamme de précocité. Globalement, les variétés montrent une grande diversité phénotypique faiblement attribuable aux facteurs géographiques (village ou zone climatique d'origine). Le cycle est un important caractère de différenciation variétale et aussi de certains types de sorgho, dont ceux à grains rouges généralement plus précoces que les sorghos à grains blancs. Deux analyses multivariées révèlent une structuration de la diversité phénotypique des variétés, expliquée, dans un premier cas, par le facteur racial et, dans le second cas, par le cycle et le type de grain avec des variétés de cycles précoces et intermédiaires, productives, présentant des grains plutôt gros et farineux se différenciant de variétés de cycles longs et très longs avec des grains de taille moyenne et à bonne vitrosité. Dans le sous-ensemble des variétés précoces et intermédiaires, les sorghos à grains rouges autres que margaritiferum constituent un groupe distinct. Les variétés à grains blancs sont moins homogènes agromorphologiquement.
\end{abstract}

Mots clés : agrobiodiversité ; Burkina Faso ; conservation des ressources ; sorgho ; variété indigène.

Thèmes : productions végétales ; ressources naturelles et environnement.

\section{Abstract \\ Phenotypic variability of local sorghum cultivars/varieties of two regions in Burkina Faso: The Boucle du Mouhoun and the Centre West}

The racial classification of 190 sorghum landraces collected in two regions of Burkina Faso and characterized for 27 agro-morphological traits shows the preponderance of Guinea varieties (96.8\%). Durra and Caudatum varieties are rare, respectively $1.6 \%$ and $0.5 \%$, whereas $1.1 \%$ of the varieties were not classified. Among the Guinea sorghums, the gambicum are more frequent than the margaritiferum which present an important variability in cycle duration. The phenotypic diversity of the landraces is high and poorly explained by geographic factors (villages or climatic zones). The differences between varieties are mainly based on vegetative duration and red-grained sorghums are generally earlier than white-grained sorghums. An initial multivariate analysis of data shows stratification based on racial aspects. In a second analysis, plant cycle and grain type characteristics discriminate two clusters: a group of early and medium cycle sorghum varieties with rather big and floury grain and a group of late cycle sorghum varieties with lower grain weight and vitreous grains. The red sorghums other than the margaritiferum form a specific cluster. In contrast, white sorghums show less homogeneity.

Key words: Burkina Faso; agrobiodiversity; sorghum grain; land varieties; resource conservation.

Subjects: natural resources and environment; vegetal productions.

Tirés à part : J. Chantereau 
a préservation des ressources phytogénétiques des espèces cultivées nécessite une connaissance approfondie de leur diversité à des fins de meilleure gestion et valorisation par l'amélioration des plantes. Au Burkina Faso, le sorgho est la première céréale alimentaire. Il occupe $48 \%$ des superficies céréalières et sa production est presque entièrement assurée par les variétés locales dominées par la race Guinea dont la variabilité est très importante (Zongo, 1991 ; Ollitrault et al., 1997). La baisse de la pluviométrie (Some, 1989), créant une inadéquation entre la durée du cycle des variétés et la durée de la saison des pluies, semble constituer un facteur d'érosion génétique. De plus, le développement de la culture cotonnière ces vingt dernières années conduit les producteurs à cultiver préférentiellement le maïs en rotation après le coton en raison de sa meilleure réponse à l'engrais par rapport au sorgho (Belem et al., 2001). De ce fait, le sorgho est de plus en plus relégué aux sols marginaux dans les régions cotonnières entraînant la disparition de certaines variétés, en particulier celles à cycle long ou sensibles à divers stress. Tous ces facteurs compromettent l'importance et la richesse variétale de cette céréale au Burkina Faso.

Pour préserver et valoriser la diversité existante, des prospections ont été réali- sées en 2003 dans trois régions agricoles du Burkina Faso : le Centre-Nord (région de climat subsahélien), la Boucle du Mouhoun et le Centre-Ouest (régions de climat nord- et sud-soudanien). Ces régions sont représentatives de la diversité des environnements agropédoclimatiques de la culture du sorgho au Burkina Faso. Toutefois, la culture du coton est prépondérante dans la Boucle du Mouhoun où les sols sont plus fertiles. Nous rapportons ici les résultats de l'étude agromorphologique réalisée sur les variétés prospectées dans les deux dernières régions, avec comme objectifs l'évaluation de la diversité de la collection et l'identification des principaux caractères qui la structurent.

\section{Matériel et méthode}

\section{Matériel végétal}

L'étude a été conduite sur 190 variétés locales prospectées en 2003, dont 81 variétés issues de 10 villages de la région de la Boucle du Mouhoun et 109 variétés provenant de 10 villages de la région du Centre-Ouest (figure 1 et tableau 1) (Vom Brocke et Simpore, 2004). Les deux régions agricoles de la prospection sont situées dans trois zones climatiques : la zone 1 (Z1) qui relève du climat subsahélien entre les isohyètes 500 et $700 \mathrm{~mm}$, la zone 2 (Z2) du climat nordsoudanien entre les isohyètes 700 et $900 \mathrm{~mm}$ et la zone 3 (Z3) du climat sudsoudanien entre les isohyètes 900 et $1100 \mathrm{~mm}$ (Guinko, 1984). Les sols en majorité peu fertiles sont de type ferrugineux tropicaux (fragilisés par l'érosion éolienne et hydrique) avec des ondulations peu marquées, présentant des sols plus profonds et argileux en bas de toposéquence où sont cultivées des variétés de sorgho plus tardives qu'en haut ou au milieu de toposéquence. Relativement à ce zonage, 17 variétés proviennent de la zone 1, 131 de la zone 2 et 42 de la zone 3).

\section{Site et dispositif expérimental}

La classification raciale selon Harlan et de Wet (1972) et la caractérisation phénotypique du matériel collecté ont été réalisées à la station de recherche de Saria $\left(2^{\circ} 09^{\prime} \mathrm{W}, 12^{\circ} 16^{\prime} \mathrm{N}\right)$ située entre les isohyètes 700 et $900 \mathrm{~mm}$.

Avec l'ajout de trois témoins communs aux expérimentations, le dispositif expérimental a été un Alpha Lattice (Patterson et Williams, 1976) à quatre répétitions, soit 12 blocs de 7 entrées par répétition pour le matériel de la Boucle du Mou-

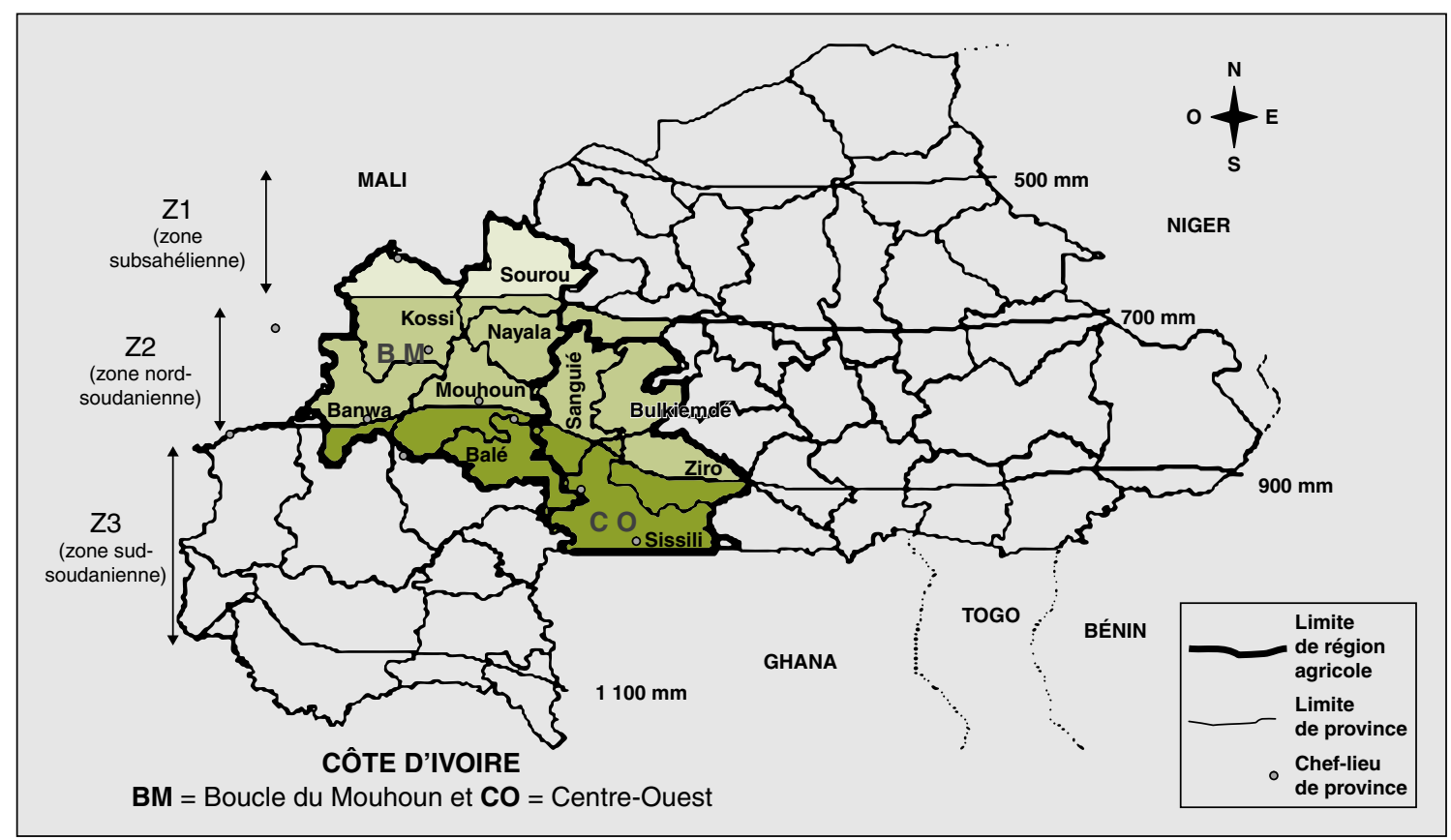

Figure 1. Provinces du Burkina Faso (administration territoriale) et zones climatiques (Guinko, 1984) concernées par l'étude.

Figure 1. Burkina Faso provinces and climatic zones covered by the sorghum collect (Guinko, 1984). 
Tableau 1. Origine géographique des variétés locales de sorgho collectées en 2003 au Burkina Faso.

Table 1. Geographic origin of collected sorghum varieties in 2003 in Burkina Faso.

\begin{tabular}{lllcl}
\hline $\begin{array}{l}\text { Région } \\
\text { agricole }\end{array}$ & Province & Village & $\begin{array}{c}\text { Nombre } \\
\text { de variétés }\end{array}$ & Zone climatique \\
\hline $\begin{array}{llll}\text { Boucle } \\
\text { du } \\
\text { Mouhoun }\end{array}$ & Sourou & Kiembara & 10 & Z1 (500-700 mm) \\
& Sourou & Kassoum & 7 & Z1 \\
& Kossi & Bomborokouy & 9 & Z2 (700-900 mm) \\
& Nayala & Biba & 10 & Z2 \\
& Mouhoun & Tchériba & 9 & Z2 \\
& Banwa & Founa & 9 & Z2 \\
& Banwa & Yasso & 8 & Z2 \\
& Banwa & Bena & 9 & Z3 (900-1 $100 \mathrm{~mm})$ \\
Centre & Balé & Sybi & 6 & Z3 \\
Ouest & Mouhoun & Kiera & 4 & Z3 \\
& Boulkiemdé & Velia & 14 & Z2 \\
& Boulkiemdé & Baonghin & 3 & Z2 \\
& Boulkiemdé & Bologo & 6 & Z2 \\
& Sanguié & Tiogo & 22 & Z2 \\
& Sanguié & Kya & 14 & Z2 \\
& Sanguié & Pouni-nord & 27 & Z2 \\
& Ziro & Tékourou & & \\
& Ziro & Lon & 6 & Z3 \\
& Sissili & Bieha & 4 & Z3 \\
& Sissili & Bourra & 5 & Z3 \\
& & & &
\end{tabular}

houn et 14 blocs de 8 entrées pour celui du Centre-Ouest. Pour chaque entrée, deux lignes de $4 \mathrm{~m}$ ont été semées en poquets le 14 juin 2003 avec un espacement de $80 \mathrm{~cm}$ entre les lignes et $40 \mathrm{~cm}$ entre les poquets sur la ligne. Un démariage à 2 plants par poquet a été effectué une dizaine de jours après la levée. L'essai a été conduit dans des conditions d'alimentation hydrique satisfaisante avec une pluviométrie utile de $646 \mathrm{~mm}$ et totale de $808 \mathrm{~mm}$.

\section{Variables observées}

Vingt-sept variables, dont 13 quantitatives, ont permis de décrire les variétés. Les variables quantitatives suivantes ont été mesurées : durée du cycle semis épiaison (Cse), hauteur de la plante mesurée à partir de sa tige principale (Hp), longueur des entre-nœuds (Len), diamètre de tige (Dt), nombre de talles utiles (Tu), longueur de panicule (Lpa), longueur (Lf) et largeur (Laf) de la troisième feuille souspaniculaire, nombre d'étages de ramification paniculaire (Ner), les poids de panicules (Ppa), de grains (Pgr), de 1000 grains (P1g). La vitrosité du grain (Vit) a été notée selon l'échelle Internatiobattage de parcelle. Les caractères qualivariables. longueur ( $\mathrm{Lgl})$ et ouverture $(\mathrm{Ogl})$ des glumes, compacité de la panicule (Cpa), forme du grain $(\mathrm{Fg})$, rotation du grain (Rgr). D'autres caractères ont été aussi observés : anthocyane du feuillage (Ant), couleur des glumes $(\mathrm{Cgl})$, couleur du grain (Cgr), taches d'anthocyane sur le grain (Tg), aristation des glumelles (Ar), adhérence des glumes (Ad) et couche brune du grain $(\mathrm{Cb})$.

Des analyses univariées ont été réalisées avec le logiciel PLABSTAT version 3A-pre (Utz, 2005). Une analyse en composantes principales (ACP) suivie d'une classification hiérarchique ascendante (CHA), d'une analyse des correspondances multiples (ACM) et d'une analyse factorielle discriminante (AFD) a été réalisée avec le logiciel XL-STAT-PRO version 7.5 19952000 (Fahmy, 1999). Pour l'ACM, les variables quantitatives ont été transformées en modalités. Seize variables peu informatives ou présentant des données manquantes ou avec une distribution anormale ont été traitées comme variables supplémentaires ou éliminées. Onze variables (Cse, Lpa, Hp, Vit, Lgl, Rgr, Lep, Pep, Ogl, Cpa, P1g) éclatées en 32 modalités ont servi à l'analyse finale.

\section{Résultats}

Board for Plant Genetic Resources, International Crops Research Institute for the Semi-Arid Tropics (1993). Les mesures ont été réalisées sur cinq plantes aléatoirement choisies par entrée à l'exception des mesures de rendement (Ppa, Pgr) faites sur la parcelle et du poids de 1000 grains (P1g) fait sur un prélèvement de tatifs étaient essentiellement des descripteurs de l'épillet : longueur (Lep) et persistance (Pep) de l'épillet pédicellé,
Les 190 variétés locales appartiennent à trois races botaniques avec 96,8\% attribuées à la race Guinea (dont 95,1\% de gambicum et $4,9 \%$ de margaritiferum), $1,6 \%$ à la race Durra et $0,5 \%$ à la race Caudatum probablement issues de la recherche (1,1\% étant des intermédiaires difficiles à classer). Par ailleurs, 96,8\% des variétés locales sont anthocyanées, $79,5 \%$ sont à glumes noires et $67,9 \%$ sont

\section{Tableau 2. Région Centre-Ouest : paramètres statistiques pour les six variables quantitatives intégralement notées.}

Table 2. Centre-West region: statistical parameters of the studied quantitative

\begin{tabular}{lccccc}
\hline Variable & Minimum & Maximum & Moyenne & F variété & CV $(\%)$ \\
\hline Cse (jour) & 67 & 112 & 89,0 & $71,3^{* *}$ & 1,8 \\
Hp (cm) & 170 & 510 & 401,8 & $7,4^{* *}$ & 6,7 \\
Lpa (cm) & 14,9 & 47,2 & 32,5 & $10,9^{* *}$ & 6,5 \\
Ner & 7 & 17 & 12,0 & $1,5^{* *}$ & 13,1 \\
P1g (g) & 12,1 & 36,4 & 24,2 & $12,5^{* *}$ & 5,6 \\
Vit & 1 & 3 & 3,1 & $16,2^{* *}$ & 10,8
\end{tabular}

Cse : cycle semis épiaison; hp : hauteur de la plante mesurée à partir de sa tige principale ; Ipa : longueur de panicule; Ner: nombre d'étages de ramification paniculaire; P1g: poids de 1000 grains ; Vit: vitrosité du grain ; CV : coefficient de variation : ** effet significatif au seuil de $1 \%$. 
à grains blancs, et enfin 80,0\% des variétés ont un grain de vitrosité très bonne à bonne (1,5-3).

Les neuf variétés Guinea margaritiferum ont des cycles végétatifs très variables (68,1-106,6 jours semis-épiaison) allant de la grande précocité à la grande tardivité. Par ailleurs, dans le matériel autre que margaritiferum, les variétés à grains rouges sont plutôt précoces avec en moyenne un cycle semis-épiaison de 84,1 jours et les variétés à grains blancs sont plus tardives avec un cycle moyen de 91,8 jours (figure 2).

Les analyses de variances ont été réalisées avec les 6 variables quantitatives (Cse, Hp, Lpa, Ner, P1g, Vit) qui ont pu être intégralement notées.

L'examen des histogrammes de fréquences a montré une distribution normale, avec une amplitude de variation importante. Par région, l'effet variétal est statistiquement significatif pour tous les caractères mesurés (tableaux 2 et 3 ). La différence entre les variétés est encore significative pour le caractère durée de cycle (Cse) à l'échelle de la zone climatique et à l'échelle du village (tableaux 4 et 5). À l'opposé, le caractère Ner est le moins souvent significatif.

Le facteur variété explique 94,6\% de la variabilité du cycle, $80,2 \%$ du poids moyen d'un grain et $75,2 \%$ de la vitrosité. Les facteurs village (20 villages) et zone climatique (3 zones) ne sont que faiblement explicatifs, sauf pour le cycle dont la détermination leur est un peu plus attribuable : respectivement pour 17,3 et $8,2 \%$.

Les corrélations entre les variables sont en général faibles. La plus forte valeur de corrélation positive est trouvée entre le cycle et la hauteur de plante $(r=0,71)$. Le cycle est négativement corrélé à la vitrosité $(r=-0,48)$ et au poids de 1000 grains $(r=-0,33)$ rendant peut-être compte d'un mauvais remplissage des grains lié à des déficits hydriques pour les variétés tardives. Ces résultats vont dans le sens de ceux de Zongo (1991) et Chantereau (1993).

Les deux premiers axes de l'ACM sur les variables Cse, Lpa, Hp, Vit, Lgl, Rgr, Lep, Pep, Ogl, Cpa, P1g expliquent 37,8\% de la variance totale. Le premier axe factoriel associe principalement des modalités du développement végétatif et discrimine les sorghos de cycle précoce, de taille réduite sans rotation du grain, aux sorghos de cycles longs, de haute taille présentant une rotation moyenne à forte du grain. Le second axe est essentiellement constitué

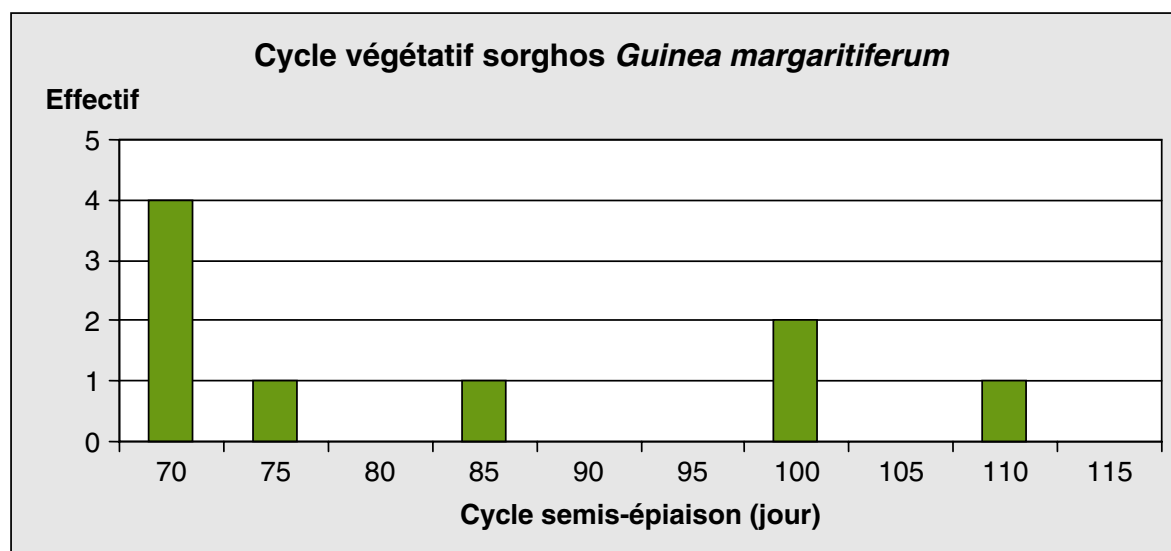

Cycle végétatif sorghos grain rouge (autres que margaritiferum)

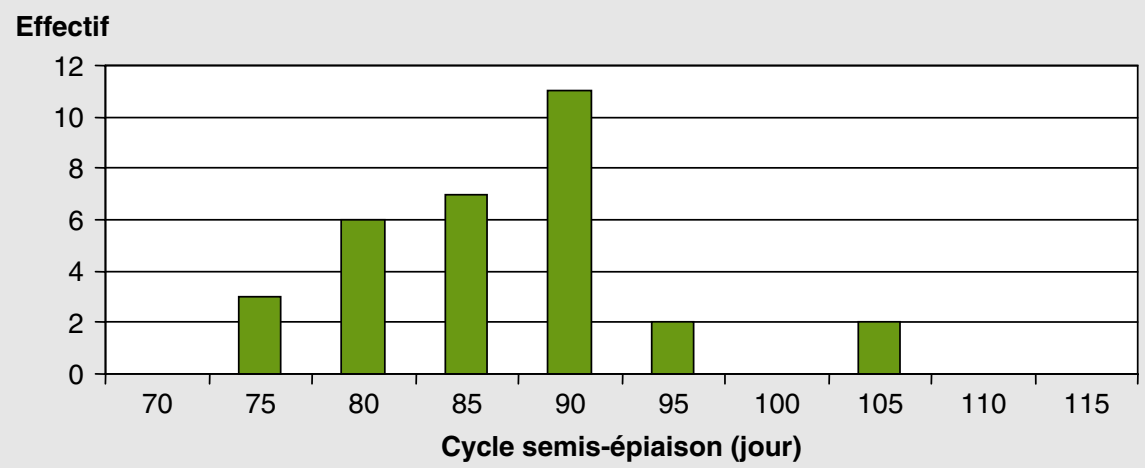

Cycle végétatif sorghos grain blanc (autres que margaritiferum)

Effectif

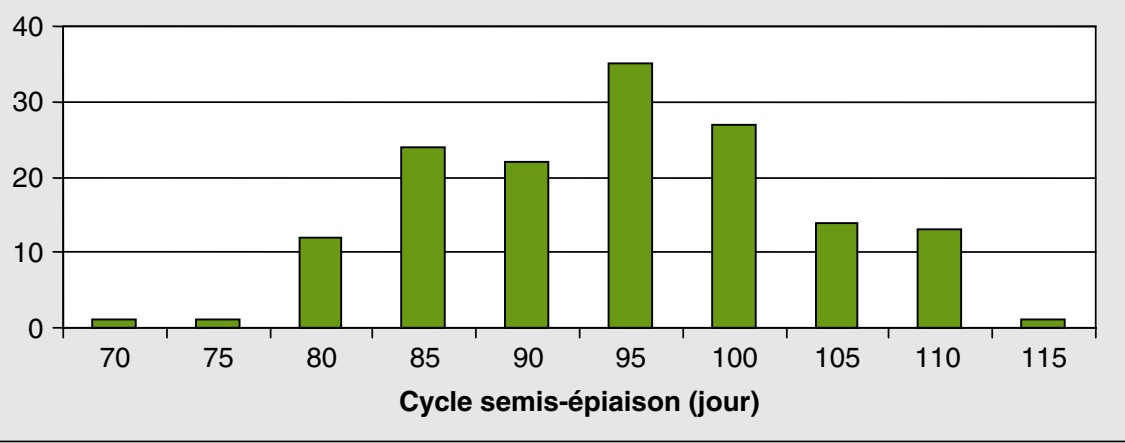

Figure 2. Histogrammes des cycles végétatifs des différents types de variétés.

Figure 2. Histogram for the vegetative cycles of different kinds of varieties.

par des modalités de caractères descriptifs de l'inflorescence.

La représentation des variétés locales dans le plan 1 x 2 de l'ACM met en évidence une structuration à caractère racial en différenciant trois grands groupes raciaux : les Guinea gambicum, les Guinea margaritiferum et les Durra (figure 3). Les variétés indéterminées se rattachent plutôt au groupe des Guinea gambicum. Cette analyse confirme que les Guinea margaritiferum sont bien distincts des autres sorghos.

Une classification hiérarchique des 190 variétés, réalisée avec le critère d'agrégation de Ward à partir des valeurs 
Tableau 3. Boucle de Mouhoun : paramètres statistiques pour les six variables quantitatives intégralement notées.

Table 3. Boucle du Mouhoun: statistical parameters of the studied quantitative variables.

\begin{tabular}{lccrrr}
\hline Variable & Minimum & Maximum & Moyenne & F variété & $\begin{array}{c}\text { CV } \\
\mathbf{( \% )}\end{array}$ \\
\hline Cse (jour) & 68 & 108 & 90,7 & $153,7^{* *}$ & 1,7 \\
Hp (cm) & 192 & 496 & 392,0 & $12,2^{* *}$ & 6,1 \\
Lpa (cm) & 21 & 52 & 36,7 & $14,3^{* *}$ & 5,8 \\
Ner & 7 & 18 & 12,1 & $1,7^{* *}$ & 12,0 \\
P1g (g) & 13,4 & 39,1 & 22,2 & $25,3^{* *}$ & 6,4 \\
Vit & 1 & 5 & 3,1 & $5,8^{* *}$ & 14,2 \\
\hline
\end{tabular}

Cse : cycle semis épiaison; hp : hauteur de la plante mesurée à partir de sa tige principale ; Ipa : Iongueur de panicule; Ner: nombre d'étages de ramification paniculaire; P1g: poids de 1000 grains ; Vit vitrosité du grain ; CV : coefficient de variation ; ${ }^{* *}$ effet significatif au seuil de $1 \%$.

Tableau 4. Mesure de l'effet variété au niveau « zones pluviométriques " pour les six variables intégralement notées.

Table 4. Performance (for the quantitative traits) of varieties pooled by rainfall zone and statistical significance of variety factor within zone.

\begin{tabular}{lccccccc}
\hline Zone & Effectif & $\begin{array}{c}\text { Cse } \\
\text { (jour) }\end{array}$ & $\begin{array}{c}\text { Hp } \\
\text { (cm) }\end{array}$ & $\begin{array}{c}\text { Lpa } \\
\text { (cm) }\end{array}$ & Ner & $\begin{array}{c}\text { P1g } \\
\text { (g) }\end{array}$ & Vit \\
\hline I & 17 & $87,5^{* *}$ & $387,9^{* *}$ & $37,9^{* *}$ & 12,1 & $22,2^{* *}$ & 3,1 \\
II & 131 & $88,7^{* *}$ & $398,1^{* *}$ & $34,1^{* *}$ & 12,0 & $23,1^{* *}$ & $3,1^{* *}$ \\
III & 42 & 95,3 & $412,2^{* *}$ & $34,2^{* *}$ & 12,2 & $22,0^{* *}$ & $3,0^{* *}$ \\
\hline
\end{tabular}

Cse : cycle semis épiaison; hp : hauteur de la plante mesurée à partir de sa tige principale ; Ipa : Iongueu de panicule; Ner: nombre d'étages de ramification paniculaire; P1g: poids de 1000 grains ; Vit : vitrosité du grain ; ** effet significatif au seuil de $1 \%$.

\section{Tableau 5. Mesure de l'effet variété au niveau « villages " pour les six variables intégralement notées.}

Table 5. Performance (for the quantitative traits) of varieties pooled by village and statistical significance of variety factor within village.

\begin{tabular}{|c|c|c|c|c|c|c|}
\hline Village & $\begin{array}{c}\text { Cse } \\
\text { (jour) }\end{array}$ & $\underset{(\mathbf{c m})}{\mathrm{Hp}}$ & $\begin{array}{l}\text { Lpa } \\
\text { (cm) }\end{array}$ & Ner & $\begin{array}{l}\text { P1g } \\
\text { (g) }\end{array}$ & Vit \\
\hline Baonghin & $81,67^{* *}$ & $389,33^{* *}$ & $33,94^{* *}$ & $11,89 * *$ & $22,3^{* *}$ & $2,83 * *$ \\
\hline Bena & $98,11 * *$ & 398,44 & 39,12 & 13,00 & $19,6 * *$ & $2,67 * *$ \\
\hline Biba & $89,70^{* *}$ & $345,55 * *$ & $32,40 * *$ & 11,80 & $22,7^{* *}$ & $3,25 * *$ \\
\hline Bieha & $102,25^{* *}$ & $434,17^{* *}$ & $30,02 * *$ & 11,25 & $20,5 * *$ & $3,00 * *$ \\
\hline Bologo & $84,06 * *$ & $391,22^{*}$ & $30,63^{* *}$ & 11,33 & $26,3^{* *}$ & $3,50 * *$ \\
\hline Bomborokouy & $86,33^{* *}$ & $367,11^{* *}$ & $36,29 * *$ & 13,11 & $21,9 * *$ & $2,94^{*}$ \\
\hline Boura & $92,53^{* *}$ & 410,40 & $31,91 * *$ & 13,13 & $23,1^{*}$ & $3,10 * *$ \\
\hline Founa & $86,44^{* *}$ & 344,42 & $35,74^{* *}$ & 11,78 & $21,2 * *$ & $3,61 * *$ \\
\hline Kassoum & $87,14^{* *}$ & $399,57^{* *}$ & $40,20 * *$ & $12,14^{* *}$ & $20,6^{* *}$ & $3,07 * *$ \\
\hline Kiembara & $87,60^{* *}$ & $339,80 * *$ & $33,22^{* *}$ & 12,50 & $23,6 * *$ & 3,05 \\
\hline Kiera & $100,25^{* *}$ & 404,75 & 35,55 & 12,75 & $21,0^{* *}$ & $2,75 * *$ \\
\hline Kya & $87,14^{* *}$ & 403,50 & $32,97^{*}$ & 12,14 & 24,0 & 2,96 * * \\
\hline Lon & $95,67 * *$ & $438,61 * *$ & $32,96 * *$ & 12,67 & $22,3^{* *}$ & $3,00 * *$ \\
\hline Pouni Nord & $88,26 * *$ & 409,23 & $31,98 * *$ & 12,19 & $24,2^{* *}$ & 2,81 \\
\hline Sybi & $91,00 * *$ & 312,83 & $28,98 * *$ & $12,00 * *$ & $23,2^{* *}$ & $3,33 * *$ \\
\hline Tchériba & $92,89 * *$ & 421,75 & $40,62^{* *}$ & 11,89 & 23,7 & $2,94 * *$ \\
\hline Tékourou & $91,25 * *$ & 409,54 & $33,98 * *$ & 11,63 & 23,7 * * & $3,19 * *$ \\
\hline Tiogo & $88,65^{* *}$ & $385,47^{* *}$ & $33,69 * *$ & 11,76 & $23,1 * *$ & $3,39 * *$ \\
\hline Velia & $89,57 * *$ & $401,95^{*}$ & $33,08 * *$ & 12,12 & 23,4 * & $3,50 * *$ \\
\hline Yasso & $99,50 * *$ & 413,63 & 37,15 & 11,63 & \multicolumn{2}{|c|}{$18,5 * 3.00$} \\
\hline
\end{tabular}

Cse : cycle semis épiaison; hp : hauteur de la plante mesurée à partir de sa tige principale ; Ipa : Iongueu de panicule; Ner: nombre d'étages de ramification paniculaire; P1g: poids de 1000 grains ; Vit : vitrosité du grain ; ** effet variétal significatif au seuil a de $5 \%$. moyennes ajustées des 6 variables de l'analyse de variance, produit le dendrogramme de la figure 4 . Un premier niveau de troncature sépare les variétés en deux ensembles de précocité différente avec respectivement 70 et 120 individus (ensembles I et II). Un découpage plus fin identifie 5 groupes (tableau 6). L'ensemble I est composé principalement des margaritiferum très précoces (groupe 1), d'un groupe de gambicum à grains blancs (groupe 2) et d'un groupe de gambicum à grains rouges (groupe 3) moyennement précoces. En plus de sa précocité, cet ensemble I se caractérise par le regroupement de sorghos à grains de faible vitrosité avec des rendements élevés en grains. L'ensemble II réunit des Guinea caractérisés par un cycle long (groupe 4) ou un cycle très long (groupe 5). Cet ensemble II se distingue aussi par des grains plutôt petits et vitreux et de fortes valeurs de hauteur de plante et de longueur paniculaire.

La caractérisation de ces groupes par l'AFD indique une probabilité associée aux valeurs de $\mathrm{F}$ hautement significative au seuil alpha de $5 \%$. La recherche des variables les plus discriminantes montre que les groupes sont établis, par ordre d'importance, sur la base de la durée du cycle, la hauteur de plante, le poids moyen d'un grain, la vitrosité et la longueur des panicules.

\section{Discussion}

La diversité raciale des sorghos cultivés dans la région Centre-Ouest et la Boucle du Mouhoun au Burkina Faso est faible, avec un important fonds génétique Guinea $(96,8 \%)$ dont une majorité de gambicum et quelques margaritiferum. La composition raciale de notre échantillon est comparable à celui de Zongo (1991) qui rapporte une proportion de $93,1 \%$ de Guinea sur l'ensemble du Burkina Faso. Cette présence dominante des Guinea s'explique par la situation biogéographique du pays, en relation avec l'histoire de la domestication du sorgho, au coeur du centre de diversité de la race Guinea, par une forme d'homogénéité culturelle concernant les pratiques agricoles et en partie par le facteur climatique : les Guinea sont adaptés aux zones les mieux arrosées comme dans la zone 3 de l'étude où seuls les Guinea sont cultivés. Il en va différemment dans d'autres pays de la sous-région. Ainsi, les Guinea ne repré- 
Tableau 6. Caractéristiques des groupes issus de la classification hiérarchique ascendante (CHA).

Table 6. Characterisation of the groups obtained from AHC.

\begin{tabular}{|c|c|c|c|c|c|c|c|c|c|c|c|c|}
\hline \multirow[t]{2}{*}{ Ensemble } & \multirow[t]{2}{*}{ Groupe } & \multirow[t]{2}{*}{ Effectif } & \multicolumn{3}{|c|}{$\begin{array}{c}\text { Race } \\
(\%)\end{array}$} & \multicolumn{2}{|c|}{$\underset{(\%)}{\text { Couleur grain }}$} & \multirow{2}{*}{$\begin{array}{c}\text { Cycle } \\
\text { semis } \\
\text { épiaison } \\
\text { (jour) }\end{array}$} & \multirow{2}{*}{$\begin{array}{c}\text { Hauteur } \\
\text { de } \\
\text { plante } \\
\text { (cm) }\end{array}$} & \multirow{2}{*}{$\begin{array}{l}\text { Longueur } \\
\text { panicule } \\
\text { (cm) }\end{array}$} & \multirow{2}{*}{$\begin{array}{c}\text { Poids } \\
1000 \\
\text { grains } \\
\text { (g) }\end{array}$} & \multirow[t]{2}{*}{$\begin{array}{l}\text { Vitrosité } \\
\text { du grain }\end{array}$} \\
\hline & & & $\mathbf{G g}^{*}$ & $\mathrm{Gm}^{* *}$ & Autres & Blanc & Rouge & & & & & \\
\hline \multirow[t]{4}{*}{$I$} & 1 & 6 & 0 & 83,3 & 16,7 & 83,3 & 16,7 & 69,8 & 273,8 & 33,7 & 15,0 & 3,5 \\
\hline & 2 & 37 & 86,5 & 0 & 13,5 & 97,3 & 2,7 & 82,7 & 371,2 & 30,7 & 25,4 & 3,0 \\
\hline & 3 & 27 & 100 & 0 & 0 & 3,7 & 96,3 & 82,3 & 374,7 & 32,7 & 25,1 & 4,7 \\
\hline & & (total 70) & & & & & & & & & & \\
\hline \multirow[t]{2}{*}{ II } & 4 & 89 & $98,990,3$ & $\begin{array}{ll}3 & 1,1\end{array}$ & 0 & 96,6 & 3,4 & 91,8 & 419,6 & 36,0 & 23,0 & 2,8 \\
\hline & 5 & $\begin{array}{c}31 \\
\text { total 120 }\end{array}$ & & 9,7 & 0 & 87,1 & 12,9 & 104,5 & 426,5 & 36,3 & 18,4 & 2,7 \\
\hline
\end{tabular}

* Gg: Guinea gambicum ; * Gm : Guinea margaritiferum.

Les valeurs affectées aux variables quantitatives sont des moyennes.

sentent que 40,2 et $7,0 \%$ des variétés cultivées au Nigeria et Tchad respectivement (Yagoua, 1994).

Le fait que le facteur village n'explique que faiblement la variabilité agromorphologique traduit l'existence d'une diversité importante des sorghos à l'intérieur d'un village, sans doute en réponse à la diversité des sols, des systèmes de culture et d'usages commune aux villages visités (par exemple, sorghos à bière, sorghos de case, sorghos de bas-fonds). La durée de

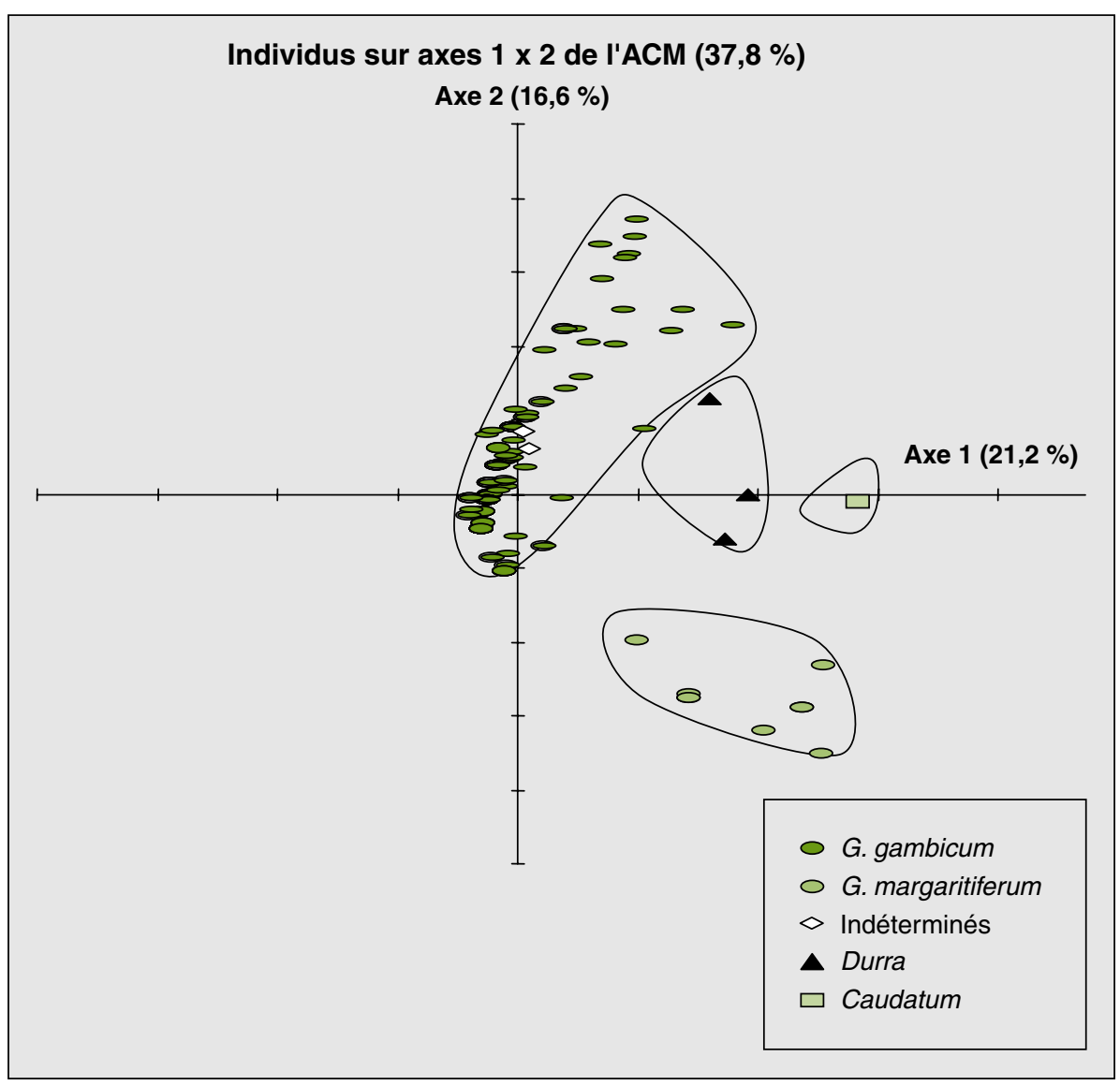

Figure 3. Structuration raciale du plan défini par les axes 1 et 2 de l'analyse des correspondances multiples (ACM).

Figure 3. Racial stratification of varieties on the first plane of MCA.

La taille plus grande de certains points gambicum dénote une superposition de points. cycle qui est une variable significativement associée aux zones climatiques serait le principal critère d'adaptation d'une variété au climat local. Néanmoins, il existe dans chaque village, un panel variétal présentant une large gamme de cycles en relation avec la complexité environnementale et des besoins traditionnels (coutumes, pharmacopée). Enfin, des échanges importants de semences entre villages de mêmes ou différentes zones climatiques concernées par notre étude existent pour des raisons de liens familiaux ou ethniques, par simple désir de tester de nouvelles variétés ou par insuffisance de stock semencier (Delaunay, 2005). Il y a sans doute là aussi une explication de la diversité variétale existant au niveau villageois.

La diversité phénotypique des sorghos est structurée par le facteur racial. Les sorghos Guinea margaritiferum se différencient des sorghos gambicum et confirment une nouvelle fois leur originalité (Degremont, 1992 ; Deu et al., 2006). En revanche, la structuration de la diversité révélée par l'analyse multivariée des seuls caractères quantitatifs est expliquée essentiellement par la durée de cycle et le type de grain. Un groupe original de sorghos est constitué par les variétés à grains rouges, autres que Guinea margaritiferum, qui se retrouvent pratiquement au sein d'un même ensemble (groupe 3) sur la base de leur précocité, de la grosseur et de la faible vitrosité de leurs grains. Ils ont également une utilisation particulière (sorghos à bière). Le groupe des sorghos à grains blancs est moins homogène, notamment par rapport à la durée de cycle. Les différences de phénologie et de pression de sélection sont sans doute à l'origine de cette structuration en 


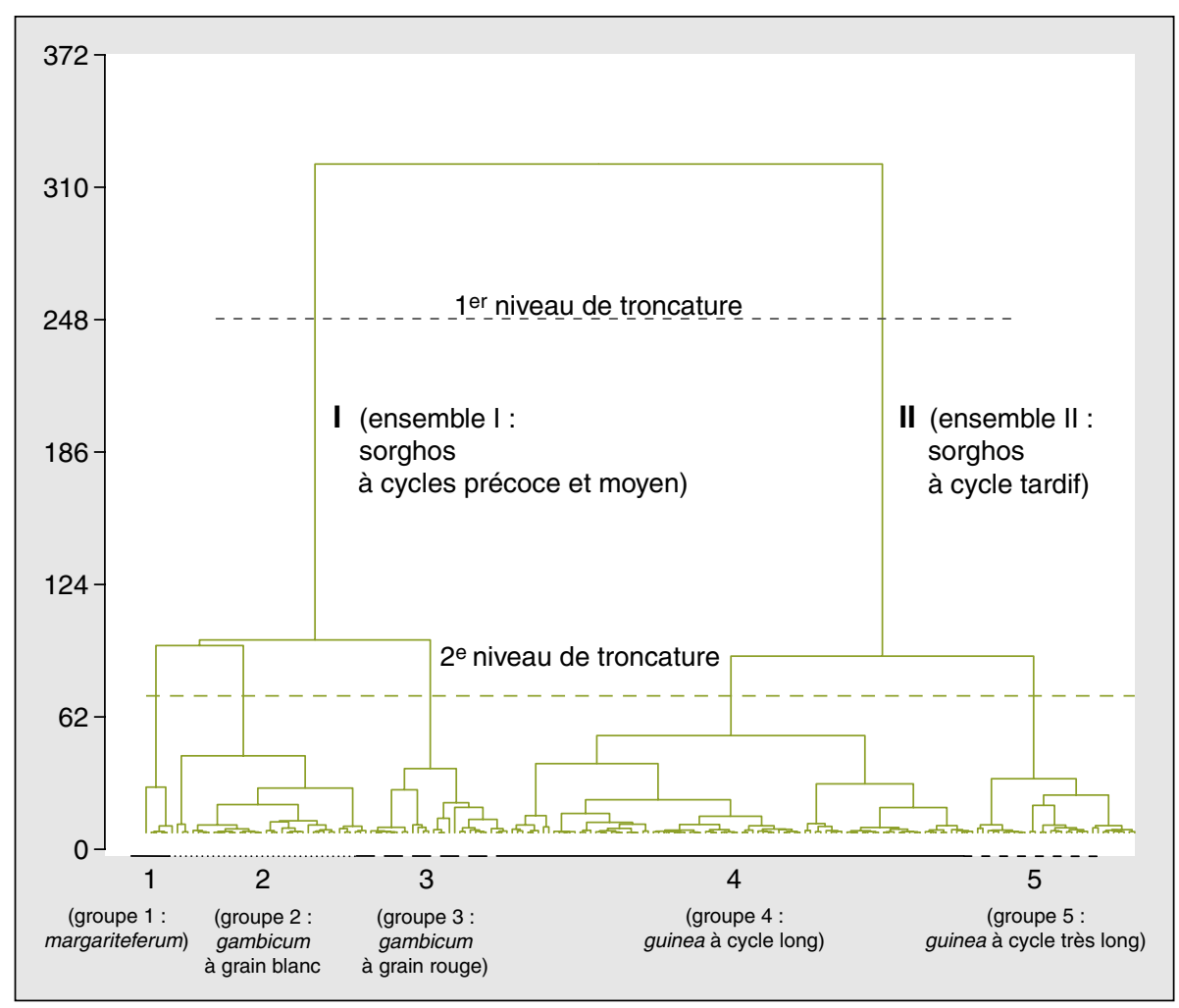

Figure 4. Classification hiérarchique ascendante $(\mathrm{CHA})$ des 190 variétés suivant le critère d'agrégation de Ward.

Figure 4. Agglomerative hierarchical classification $(\mathrm{AHC})$ of 190 varieties according to Ward's aggregative criterium.

plusieurs ensembles entre lesquels les brassages génétiques semblent être limités. Chacun de ces groupes constitue donc une entité évolutive spécifique.

En termes de conservation des ressources génétiques, notre étude montre l'importance de l'implication paysanne à l'échelle villageoise dans la gestion de la diversité des sorghos au Burkina Faso et elle souligne le besoin d'accorder une attention particulière au groupe des sorghos à grains rouges utilisés traditionnellement pour la bière. L'utilisation de marqueurs moléculaires permettra une analyse plus fine des facteurs d'évolution de la diversité génétique et une utilisation mieux raisonnée du germoplasme local dans les programmes d'amélioration. L'enjeu est de maintenir et valoriser une diversité variétale et génétique originale maîtrisée par les agriculteurs, bien adaptée aux contraintes environnementales et capable de répondre à leur évolution. La culture du sorgho continuera alors à jouer son rôle dans la sécurisation et la diversi- fication de la production céréalière du pays.

\section{Remerciements}

Albert Barro, Dominique Compaoré, Sansan Da, David Kambou, Gnissa Konaté, Grégoire Pale, Jacob Sanou, Jean-Baptiste Taonda (Inera, Burkina Faso), Bettina Hausmann (International Crops Research Institute for the Semi-Arid Tropics, ICRISAT, Niger).

\section{Références}

Belem C, D'herbes JM, Beauval V. Projet préservation de l'agrobiodiversité du sorgho au Mali et au Burkina Faso. Rapport de faisabilité. Paris: Fond français pour I'environnement mondial (FFEM) ; Centre de coopération internationale en recherche agronomique pour le développement (Cirad) ; Institut de l'environnement et des recherches agricoles (Inera). Institut d'économie rurale (IER), 2001.
Chantereau J. Étude de I'hétérosis chez le sorgho [Sorghum bicolor (L.) Moench] par l'exploitation d'écotypes et l'analyse de leurs divergences. Thèse de doctorat en sciences, université Paris XI Orsay (France), 1993.

Degremont I. Évaluation de la diversité génétique et du comportement en croisement de sorghos [Sorghum bicolor (L.) Moench] de la race Guinea au moyen de marqueurs enzymatiques et morphologiques. Thèse de doctorat, université Paris XI, Orsay (France), 1992.

Delaunay S. L'importance des modes et des stratégies d'échange de semences dans les systèmes semenciers traditionnels de sorgho au Burkina Faso. Stage d'initiation à la recherche. Esitpa-Cirad-Inera, 2005.

Deu M, Rattunde F, Chantereau J. A global view of genetic diversity in cultivated sorghums using a core collection. Genome 2006 ; $49: 168-80$.

Fahmy T. XLSTAT-PRO. Paris (France) : Addinsoft, 1999.

Guinko S. Végétation de la Haute-Volta. Thèse de doctorat, université de Bordeaux III, 1984.

Harlan JR, de Wet JMJ. A simplificated classification of cultivated sorghum. Crop Sci 1972 ; $12: 172-6$.

International Board For Plant Genetic Ressources (IBPGR), International Crops Research Ins titute for the Semi-Arid Tropics (ICRISAT). Descriptors for sorghum, [Sorghum bicolor (L.) Moench]. Rome (Italie); Patancheru (India): IBPGR ; ICRISAT, 1993.

Ollitrault P, Noyer JL, Chantereau J, Glaszmann JC. Structure génétique et dynamique des variétés traditionnelles de sorgho au Burkina Faso. Actes du colloque "Gestion des ressources génétiques des plantes en Afrique des savanes ". Bamako (Mali): Institut d'économie rurale (IER); Bureau des ressources génétiques (BRG); Solagral, 1997.

Patterson HD, Williams ER. A new class of resolvable incomplete block designs. Biometrica $1976 ; 63: 83-92$

Somé L. Diagnostic agroclimatique du risque de sécheresse au Burkina Faso. Etude de quelques techniques agronomiques améliorant la résistance pour les cultures de sorgho, mil, maïs. Thèse de doctorat, université Montpellier STL (France), 1989.

Utz HF. PLABSTAT (Version 3A-pre of 2005-0816), a computer program for statistical analysis of plant breeding experiments. Stuttgart (Germany): Institute of Plant Breeding, Seed Science and Population Genetics, University of Hohenheim, 2005.

Vom Brocke K, Simpore A. Les sorghos du village. Rapport de collecte des variétés locales de sorgho dans 30 villages au Burkina Faso. Ouagadougou : Institut de l'environnement et des recherches agricoles (Inera), 2004.

Yagoua ND. Caractérisation du sorgho pluvial, [Sorghum bicolor (L.) Moench], de la zone soudanienne du Tchad. Atelier de formation sur les variétés locales de sorgho, 10-14 octobre 1994, Samanko (Mali). 1994.

Zongo JD. Ressources génétiques des sorghos [Sorghum bicolor (L.) Moench] du Burkina Faso : évaluation agromorphologique et génétique Thèse de doctorat d'Etat université d'Abidjan (Côte d'Ivoire), 1991. 